\title{
Search for binary central stars of the SMC PNe
}

\author{
M. Hajduk ${ }^{1}$, M. Gładkowski ${ }^{1,2}$, and I. Soszyński ${ }^{3}$ \\ ${ }^{1}$ Nicolaus Copernicus Astronomical Center, ul. Rabiańska 8, 87-100 Toruń, Poland \\ e-mail: Marcin.Hajduk@astri.uni.torun.pl \\ 2 Centrum Astronomii UMK, ul. Gagarina 11, 87-100 Toruń, Poland \\ 3 Warsaw University Observatory, Al. Ujazdowskie 4, 00-478 Warszawa, Poland
}

Received 11 April 2013 / Accepted 29 October 2013

\begin{abstract}
Aims. The Optical Gravitational Lensing Experiment (OGLE), originally designed to search for microlensing events, provides a rich and uniform data set suitable for studying the variability of certain types of objects. We used the OGLE data to study the photometry of central stars of planetary nebulae (PNe) in the Small Magellanic Cloud (SMC). In particular, we searched for close binary central stars with the aim to constrain the binary fraction and period distribution in the SMC. We also searched for PNe mimics and removed them from the PNe sample.

Methods. We identified 52 counterparts of PNe in the SMC in the I-band images from the OGLE-II and OGLE-III surveys. We analysed the time-series photometry of the PNe. Spectra of the photometric variables were obtained to constrain the nature of the objects or search for additional evidence for binarity.

Results. Eight variables were found. Of these, seven objects are PNe mimics, including one symbiotic star candidate. One close binary central star of PN with a period of 1.15 or 2.31 day was discovered. The obtained binary fraction for the SMC PNe and the observational biases are discussed in terms of the OGLE observations.
\end{abstract}

Key words. planetary nebulae: general - binaries: general - binaries: symbiotic - Magellanic Clouds - binaries: eclipsing

\section{Introduction}

Large-scale photometric surveys provide an excellent opportunity to improve our understanding of the nature of the planetary nebula (PN) phenomenon. Using large photometric surveys allowed researchers to monitor the brightness of hundreds of central stars of planetary nebulae (CSPNe, Miszalski et al. 2009a; Lutz et al. 2010; Jones et al. 2011; Hajduk et al. 2011). The number of known binary CSPNe was more than doubled in recent years (De Marco 2011). The fration of close binary CSPNe was established as $12-21 \%$ on the basis of the Optical Gravitational Lensing Experiment (OGLE) photometry in the Galactic bulge (Miszalski et al. 2009a), consistent with the 10-15\% fraction given by Bond (2000).

The growing number of binary CSPNe made it possible to compare the period distribution with the population synthesis model predictions and to study the influence of the common envelope (CE) phase of the binary evolution on the morphology of the nebulae (De Marco 2009; Miszalski et al. 2009a,b; Boffin et al. 2012).

Here, we present OGLE-II and OGLE-III observations of the Small Magellanic Cloud (SMC) PNe (Udalski et al. 1997, 2008). We report the discovery of one binary nucleus. Seven objects are confirmed to be PN mimics, including a symbiotic mira candidate.

^ Table 4 is only available at the CDS via anonymous ftp to cdsarc.u-strasbg.fr (130.79.128.5) or via http://cdsarc.u-strasbg.fr/viz-bin/qcat?]/A+A/561/A8

\section{PNe sample}

SMC PNe were selected using the SIMBAD database. We made use of the object type and coordinate search criteria and found 108 objects. The list includes PNe discovered by Lindsay (1961), Sanduleak et al. (1978), Sanduleak \& Pesch (1981), Morgan \& Good (1985), Morgan (1995), and Meyssonnier (1995) by means of objective-prism plates or long-slit spectroscopy. Jacoby \& De Marco (2002) used narrow-band imaging, but subsequently confirmed their sample with spectroscopic follow-up. Jacoby (1980) used on-line/off-line filter photography. Their sample was spectroscopically verified by Boroson \& Liebert (1989), who showed that $30 \%$ of Jacoby's PNe candidates could not be confirmed or have other explanations.

We supplemented our list with nine PNe confirmed by Morgan (1995) and one from Sanduleak et al. (1978), which were missing from the Simbad database. We also included one object from Jacoby \& De Marco (2002), classified as a Heburning asymptotic giant branch (AGB) star in the Simbad database. The total sample comprises 119 objects.

The objects were identified by visual comparison of the finding charts available in the literature with the OGLE-II and OGLE-III $I$-band reference images. In addition, we observed 27 objects in the SMC with the $1.0 \mathrm{~m}$ telescope at the South African Astronomical Observatory (SAAO) with the $\mathrm{R}$ and $\mathrm{H} \alpha$ filters. No $\mathrm{H} \alpha$ emission was identified in three cases (Jacoby SMC 12, Jacoby SMC 15 and [JD2002]3). Jacoby SMC 12 and Jacoby SMC 15 were misclassified as PNe (Boroson \& Liebert 1989). [JD2002] 3 shows faint, extended nebula, probably below the sensitivity of our $\mathrm{H} \alpha$ image (Jacoby \& De Marco 2002). 
Table 1. List of PNe identified in the OGLE-II and OGLE-III fields in the SMC.

\begin{tabular}{|c|c|c|c|c|c|c|c|c|}
\hline Name & RA & Dec & $V$ & $I$ & $N_{\mathrm{I}}$ & Ref & Diameter $(\operatorname{arcsec})$ & Other names \\
\hline LHA $115-\mathrm{N} 4$ & 03421.99 & -731321.4 & 16.809 & 18.464 & 700 & $(1)$ & $0.54(11)$ & LIN 16, SMP 3 \\
\hline LHA $115-N 5$ & 04121.68 & -724516.7 & 15.805 & 17.224 & 705 & (1) & & LIN 32, SMP 5, (MA93) 23 \\
\hline LHA $115-N 6$ & 04127.75 & -734706.5 & 15.916 & 16.942 & 1213 & (1) & $\ldots(10)$ & SMP 6, LIN 33, (MA93) 29 \\
\hline Jacoby SMC 1 & 04228.15 & -732055.1 & 18.230 & 19.598 & $603+671$ & $(2)$ & & SMP 7, (MA93) 39 \\
\hline (MA93) 44 & 04309.40 & -730803.7 & 19.803 & 20.487 & $307+655$ & (6) & & \\
\hline LHA $115-N 7$ & 04325.32 & -723818.9 & 16.212 & 17.365 & 705 & (1) & $0.41 \times 0.38(10)$ & LIN 43, SMP 8, (MA93) 49 \\
\hline MGPN SMC 6 & 04425.73 & -735139.3 & 20.417 & 20.535 & 1143 & (7) & & (MA93) 73 \\
\hline (JD2002) 1 & 04512.10 & -731858.0 & 20.487 & 20.445 & $308+682$ & (9) & & \\
\hline LIN 66 & 04520.65 & -732410.3 & 17.494 & 19.155 & $399+697$ & (5) & $1.20(10)$ & J3, SMP 9, (MA93) 98 \\
\hline LIN 71 & 04527.43 & -734214.5 & 15.659 & 14.505 & 1215 & (5) & $0.27(10)$ & (MA93) 104, J4 \\
\hline (JD2002) 2 & 04536.66 & -732404.3 & 17.993 & 17.881 & $322+697$ & (9) & & \\
\hline$(J D 2002) 5$ & 04739.99 & -723903.0 & 19.443 & 19.425 & 753 & (9) & & \\
\hline LHA $115-\mathrm{N} 29$ & 04836.55 & -725800.9 & & 16.715 & $298+723$ & (1) & $0.78 \times 0.66(10)$ & SMP 11, LIN 115, (MA93) 241, J8 \\
\hline$(J D 2002) 6$ & 04908.24 & -730223.6 & 19.336 & 19.290 & $337+722$ & (9) & & \\
\hline Jacoby SMC 9 & 04920.11 & -731735.3 & 19.817 & 19.881 & $334+30$ & (3) & & (MA93) 290 \\
\hline SMP SMC 12 & 04921.10 & -735258.7 & 18.536 & 17.983 & 354 & (4) & & (MA93) 291 \\
\hline$(\mathrm{JD} 2002) 7$ & 04935.44 & -732633.7 & 19.835 & 18.868 & $325+545$ & (9) & & \\
\hline (M95) 3 & 04947.47 & -741440.0 & 17.663 & 17.859 & 694 & $(8)$ & & \\
\hline LHA $115-\mathrm{N} 38$ & 04951.65 & -734421.4 & 15.382 & 16.913 & 547 & (1) & & \\
\hline LHA $115-\mathrm{N} 40$ & 05035.09 & -734258.1 & 16.500 & 18.095 & 700 & (1) & $0.83(10)$ & \\
\hline (MA93) 406 & 05052.38 & -734455.2 & 18.574 & 19.751 & 691 & (6) & & \\
\hline LHA $115-\mathrm{N} 43$ & 05107.38 & -735737.6 & 15.341 & 16.444 & 709 & (1) & $0.32(11)$ & SMP 15, LIN 174 (MA93) 433 \\
\hline (JD2002) 12 & 05107.82 & -731206.4 & 18.330 & 17.349 & $337+722$ & (9) & $1.15 \times 1.40(11)$ & \\
\hline LHA $115-\mathrm{N} 42$ & 05127.17 & -722611.7 & 16.741 & 16.922 & 753 & (1) & $0.33 \times 0.30(11)$ & LIN 179, SMP 16, J14, (MA93) 467 \\
\hline LHA $115-\mathrm{N} 47$ & 05158.13 & -732031.3 & 16.158 & 16.626 & $339+713$ & (1) & $0.14(10)$ & LIN 196, (MA93) 519, J19, SMP 18 \\
\hline LIN 239 & 05311.13 & -724507.5 & 16.432 & 17.873 & $321+752$ & (5) & $0.59(10)$ & J20, SMP 19, (MA93) 652 \\
\hline (MA93) 891 & 05559.42 & -721400.8 & 19.274 & 20.314 & 712 & (6) & & \\
\hline LIN 302 & 05619.48 & -720658.4 & 17.609 & 17.792 & 721 & (5) & $1.39 \times 1.28(10)$ & MGPN 8, (MA93) 933 \\
\hline LIN 305 & 05630.77 & -722702.1 & 17.181 & 18.023 & $209+617$ & (5) & & (MA93) 943, SMP 21 \\
\hline (JD2002) 17 & 05652.53 & -722102.3 & 19.968 & 19.129 & 661 & (9) & & \\
\hline LIN 343 & 05842.32 & -725659.8 & 16.910 & 18.387 & $307+713$ & (5) & $0.66 \times 0.60(10)$ & J26, SMP 23, (MA93) 1088 \\
\hline LHA $115-\mathrm{N} 68$ & 05843.04 & -722716.2 & & 18.920 & $288+712$ & (1) & & (MA93) 1091, LIN 339, MGPN 9 \\
\hline LHA $115-\mathrm{N} 70$ & 05916.12 & -720200.0 & 16.016 & 16.916 & 721 & (1) & $0.38(10)$ & SMP 24, (MA93) 1136, LIN 347 \\
\hline (JD2002) 19 & 05929.37 & -733905.9 & 20.943 & 20.641 & 631 & (9) & & \\
\hline LIN 357 & 05940.51 & -713815.1 & 17.856 & 18.581 & 704 & (5) & & SMP 25, (MA93) 1159 \\
\hline (JD2002) 20 & 10015.11 & -721640.7 & 20.567 & 21.140 & 541 & (9) & & \\
\hline (JD2002) 23 & 10200.91 & -725902.0 & 20.560 & 20.783 & $313+463$ & (9) & & \\
\hline (MA93) 1438 & 10404.69 & -713724.9 & 20.320 & 20.421 & 669 & (6) & & \\
\hline LIN 430 & 10417.90 & -732151.1 & 18.124 & 19.151 & 737 & (5) & $0.61 \times 0.57(10)$ & SMP 26, (MA93) 1454 \\
\hline (MA93) 1709 & 10951.84 & -732050.6 & 19.457 & 18.657 & 735 & (6) & & \\
\hline (MA93) 1714 & 11004.86 & -724527.5 & 19.342 & 18.411 & 675 & (6) & & \\
\hline SMP SMC 34 & 11210.86 & -712650.6 & 18.584 & 19.771 & 648 & (4) & $0.71 \times 0.69(10)$ & (MA93) 1757 \\
\hline (MA93) 1762 & 11240.25 & -725346.8 & 20.109 & 20.309 & 90 & (6) & $1.45 \times 1.26(10)$ & \\
\hline LHA 115-N 87 & 12110.65 & -731434.8 & 15.553 & 16.758 & 637 & (1) & $0.45(10)$ & LIN 532, SMP 27, (MA93) 1884 \\
\hline LIN 536 & 12411.85 & -740232.2 & 17.589 & 17.716 & 625 & $(5)$ & $0.31(11)$ & SMP 28 \\
\hline
\end{tabular}

Notes. Column $N_{\mathrm{I}}$ gives the number of the OGLE-II (when available) and OGLE-III observations.

References. (1) Henize (1956); (2) Sanduleak et al. (1978); (3) Jacoby (1980); (4) Sanduleak \& Pesch (1981); (5) Lindsay (1961); (6) Meyssonnier \& Azzopardi (1993); (7) Morgan \& Good (1985); (8) Morgan (1995); (9) Jacoby \& De Marco (2002); (10) Stanghellini et al. (2003); (11) Shaw et al. (2006).

We identified 52 objects in the OGLE reference images in total out of 119 candidates (Tables 1 and 2). Though all the $\mathrm{PNe}$ in the sample were spectroscopically confirmed, classification of eight objects was ambiguous (Table 2). [MA93] 22 was classified as a PN (Meyssonnier 1995) even though it shows a strong continuum and a very weak [O III] $5007 \AA$ line. Haberl \& Sasaki (2000) classified it as a Be/X-ray binary. The PN nature of LIN 34 and Jacoby SMC 7 was questioned by Morgan (1995). The former object was classified as a B0-5V star by Evans et al. (2004). Jacoby SMC 23 is a young stellar object (YSO) candidate (Bolatto et al. 2007) and Jacoby SMC 24 is probably an H II region (Boroson \& Liebert 1989).
Henize \& Westerlund (1963) classified LHA 115-N9, LHA $115-$ N61, and LHA 115-N68 as diffuse nebulae. But we classify LHA $115-\mathrm{N} 68$ as a PN, since it shows a prominent [O III] $5007 \AA$ line (Leisy \& Dennefeld 2006). [JD2002] 11 was classified as an extreme AGB star candidate on the basis of the infrared colour-magnitude diagram (Boyer et al. 2011).

Seven known PN mimics show photometric variability. We present here the spectroscopy and photometry of these objects in addition to the genuine PNe to study their characteristics (which may be of interest to other research groups) in an endeavour to understand their nature, before ultimately rejecting them from the PN sample. 
Table 2. List of the PNe mimics showing photometric variability.

\begin{tabular}{lcccccccc}
\hline \hline Name & RA & Dec & $V$ & $I$ & $N_{\mathrm{I}}$ & Ref & Diameter [arcsec] & Other names \\
\hline (MA93) 22 & 04109.13 & -730647.1 & 17.796 & 17.764 & $304+691$ & $(4)$ & & \\
LIN 34 & 04215.77 & -725955.4 & 16.441 & 16.450 & $315+537$ & $(3)$ & & (MA93) 37 \\
LHA 115-N 9 & 04336.69 & -730226.9 & 15.529 & 15.883 & $317+698$ & $(1)$ & & (MA93) 54, LIN 45 \\
Jacoby SMC 7 & 04805.36 & -730905.9 & 19.278 & 18.947 & $324+722$ & $(2)$ & & \\
(JD2002) 11 & 05052.66 & -725216.7 & 21.263 & 19.562 & $333+722$ & $(6)$ & & (MA93) 852 \\
Jacoby SMC 23 & 05530.42 & -725021.9 & 18.358 & 17.835 & $295+713$ & $(5)$ & $\ldots$ (7) & (MA93) 955 \\
Jacoby SMC 24 & 05639.40 & -723907.2 & 17.920 & 16.619 & $295+713$ & $(2)$ & & \\
\hline
\end{tabular}

References. (1) Henize \& Westerlund (1963); (2) Boroson \& Liebert (1989); (3) Morgan (1995); (4) Haberl \& Sasaki (2000); (5) Bolatto et al. (2007); (6) Boyer et al. (2011); (7) Stanghellini et al. (2003).

Table 3. Spectroscopic observations of objects showing photometric variability.

\begin{tabular}{lccl}
\hline \hline Date & Telescope & Exptime [s] & Name \\
\hline $2012-09-17$ & SALT & 120 & Jacoby SMC 24 \\
$2012-09-19$ & SALT & $120+1200$ & Jacoby SMC 23 \\
$2012-09-19$ & SALT & $2 \times 120+1200$ & Jacoby SMC 1 \\
$2012-10-10$ & SALT & $120+1200$ & Jacoby SMC 1 \\
$2012-11-25$ & SALT & 900 & Jacoby SMC 7 \\
$2012-11-26$ & SALT & 900 & [JD2002] 11 \\
$2013-07-17$ & Radcliffe & $2 \times 1800$ & LHA 115-N 61 \\
$2013-07-21$ & Radcliffe & $3 \times 1200$ & LIN 34 \\
$2013-07-21$ & Radcliffe & $2 \times 1200$ & [MA93] 22 \\
\hline
\end{tabular}

\section{Observations}

We used the $V$ - and $I$-band photometry of the SMC collected by the OGLE-III project. OGLE-II photometry was available for some of the objects as well. The $V$-band photometry was obtained only intermittently, while I-band observations were made frequently. The $I$-band filter had a wide long-wavelength wing, quite different from the sharp drop of transmission at about $9000 \AA$ of the standard I-bandpass (Udalski et al. 2002). The data were taken only under good seeing conditions. The median seeing during the observations of the SMC was equal to 1 .'.2 (Udalski et al. 2008).

The photometry of some objects is affected by systematic effects, which hampers the time-series analysis. This problem was previously reported by Miszalski et al. (2009a) for CSPNe suffering from strong nebular contamination. We examined OGLE I-band images of our objects. Only two of them are clearly resolved: LHA 115-N 9 and LHA 115-N 61, both classified as diffuse nebulae (Sect. 2). Some other objects have full width at half maximum (FWHM) that is somewhat larger than that of the field stars.

Eight objects were found to be variable. We performed spectroscopic observations of five variables with the Southern African Large Telescope (SALT), and three variables were observed with the Radcliffe $1.9 \mathrm{~m}$ telescope at SAAO (Table 3). The SALT low-resolution spectra were obtained with the $900 \mathrm{l} / \mathrm{mm}$ grating. The slit was 1 arcsec wide and 8 arcmin long. The spectral coverage was 4350-7400 $\AA$ with the spectral resolution of about $\lambda / \Delta \lambda=6000$ at the central wavelength. A 2 arcsec wide slit and the $3001 / \mathrm{mm}$ grating was used for the $1.9 \mathrm{~m}$ telescope, resulting in the spectral resolution of about 2500 at the central wavelength. The spectral coverage was set to 3500-7500 $\AA$. All the spectra were flux calibrated.

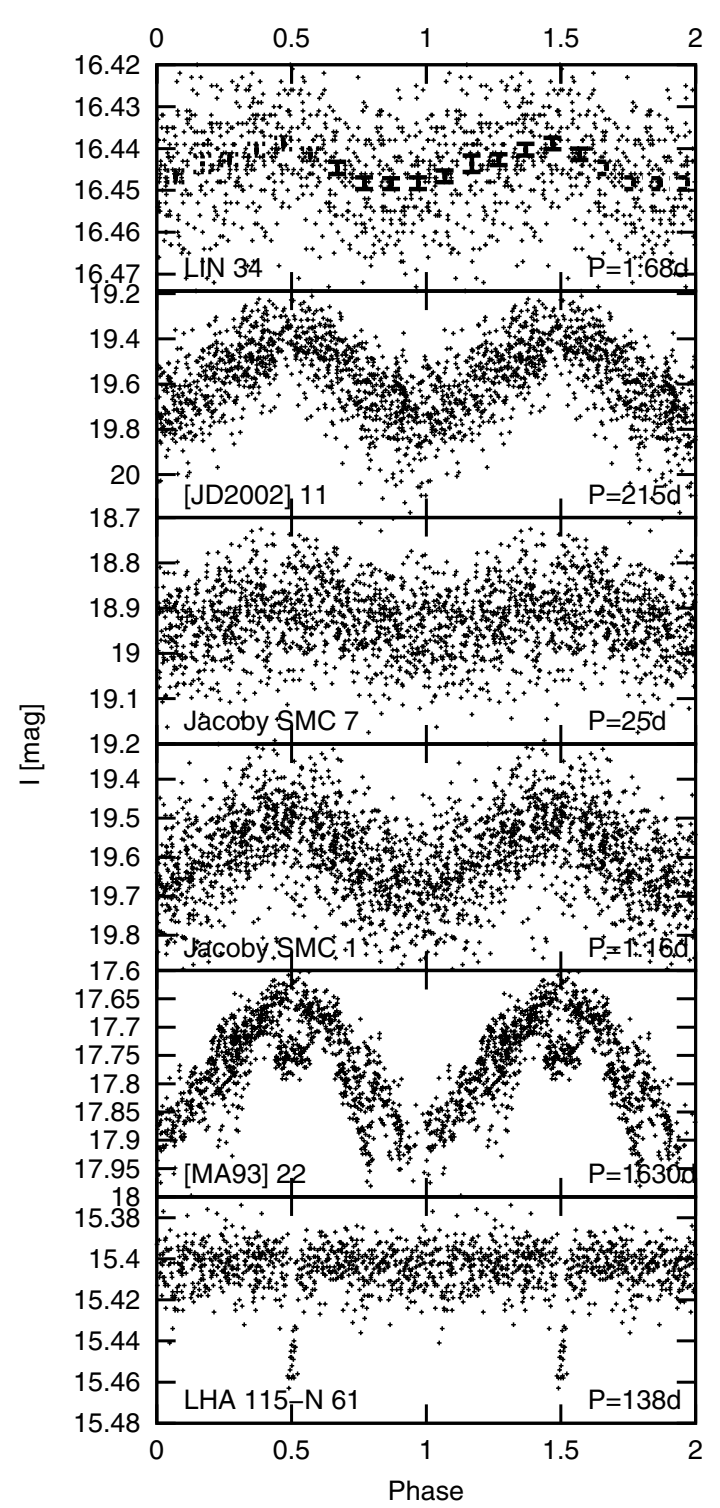

Fig. 1. Phased OGLE $I$-band light curves of the SMC periodic variables.

\section{Results}

We analysed the OGLE-II and OGLE-III $I$-band photometry using the program Period04 (Lenz \& Breger 2005) and the analysis of variance (ANOVA) method (Schwarzenberg-Czerny 1996). We identified six periodic variables (including one with a period of about 4.5 years) and two irregular variables. The phased light curves of the all periodic variables are presented in Fig. 1. 


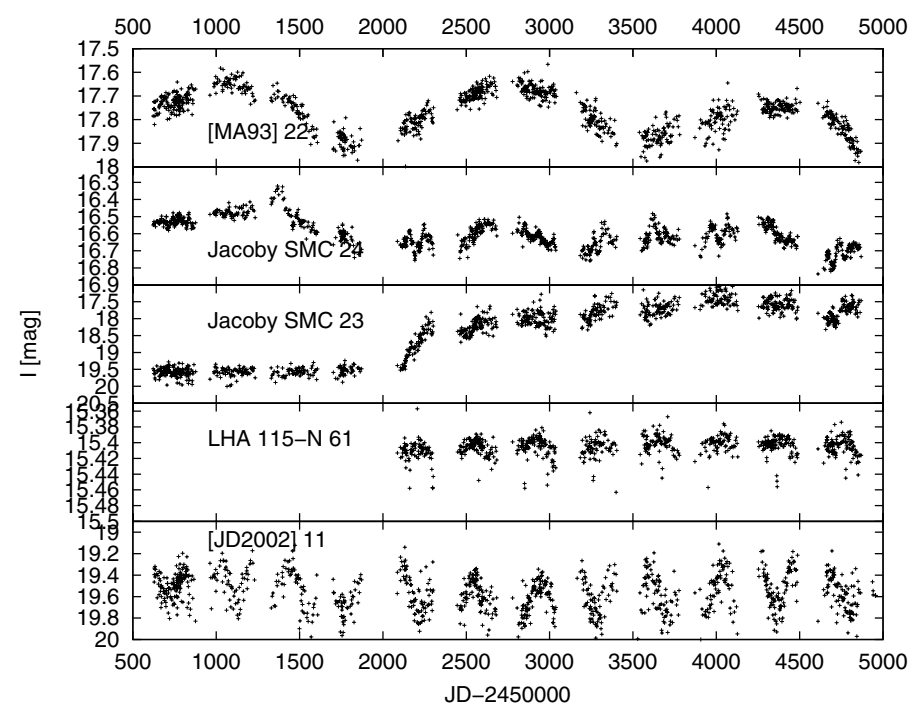

Fig. 2. OGLE-II and -III photometry of the variable objects with periods long enough to be readily presented.

The irregular light curves and periodic light curves with periods long enough to be legible are shown in Fig. 2. The spectra of all the variable objects but [MA93] 22 are presented in Fig. 3. All the spectra are available at the CDS (Table 4).

Out of the total sample of eight variable objects detected, we considered seven objects to be PNe mimics. They have previously been suggested to be PNe mimics or were considered as objects of uncertain nature. One remaining (periodic) variable is most likely a genuine binary CSPN.

\subsection{Variable stars}

LIN 34 has a period of $1.67886 \pm 0.00007$ day (Fig. 1). It is catalogued as a PN, but Meyssonnier \& Azzopardi (1993) classified it as a very low excitation (VLE) object. The spectrum of LIN 34 is characterised by strong continuum and lack of obvious [O III] $5007 \AA$ emission (Fig. 3). The observed wavelength of the emission lines corresponds to a radial velocity of about $-30 \mathrm{~km} \mathrm{~s}^{-1}$, thus LIN 34 appears to be a pulsating Galactic halo Be star (Greenstein \& Sargent 1974).

[MA93] 22 was classified as a PN by Meyssonnier (1995), although its spectrum revealed a strong continuum and a very weak [O III] $5007 \AA$ line. The object shows variability on a timescale of about 1630 days (Fig. 2). Three maxima were covered by the OGLE-II and -III phase observations. The last maximum is fainter than the first two maxima by about 0.1 mag, which affected the appearance of the phased light curve of the object (Fig. 1). The object showed only a weak $\mathrm{H} \alpha$ emission in a very noisy $1.9 \mathrm{~m}$ telescope spectrum, not shown in Fig. 3. [MA93] 22 was proposed as the optical counterpart of an X-ray binary afterwards (Haberl \& Sasaki 2000). It is clearly detected in the 2MASS $K$ band, but not detected in the $J$ and $H$ bands. The near-IR emission lines may significantly contribute to the brightness of the object in the $K$ band (Naik et al. 2012). The optical variability could correspond to the orbital period of the binary.

[JD 2002] 11 has a period of $215.3 \pm 0.2$ days (Fig. 1). The [O III] $5007 \AA$ emission line is detected in the spectrum, exceeding the $\mathrm{H} \beta$ line in intensity (Fig. 3). [JD 2002] 11 probably is a symbiotic system. The 215.3 day period may be due to the pulsation of the cool component, while the hot component is

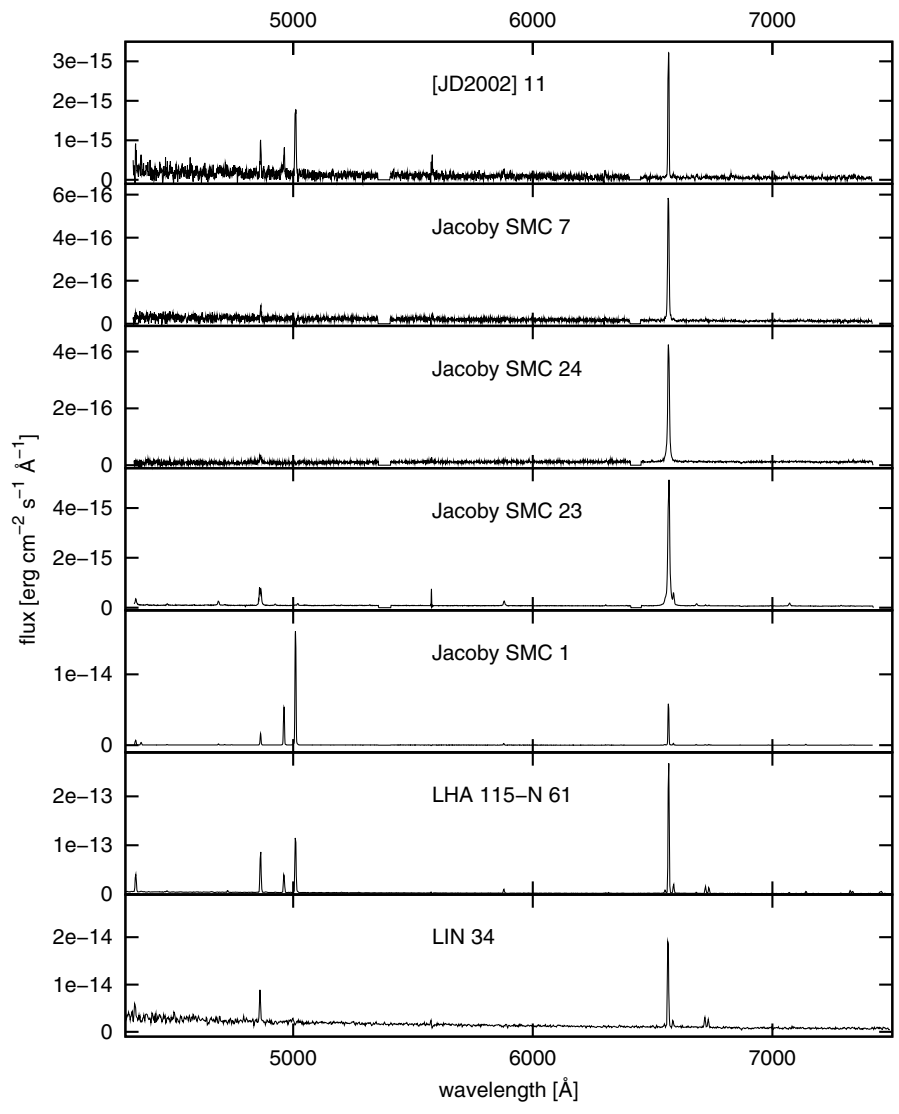

Fig. 3. Spectra of the variable objects.

responsible for the ionization of the nebula. However, the quality (and wavelength range) of the spectrum did not allow us to unveil the spectral signature of the cool component.

Near-infrared photometry allows one to separate genuine PNe from symbiotic miras (Schmeja \& Kimeswenger 2001). $\mathrm{PNe}$ are located in a well-defined region in the IJK twocolour diagram. We searched for the Two Micron All Sky Survey (2MASS) photometry of all the PNe identified in the SMC (Skrutskie et al. 2006). Infrared colours confirm that [JD 2002] 11 is a symbiotic mira and not a PN (Fig. 4). If the classification is correct, then [JD 2002] 11 would be only the eight symbiotic star discovered in the SMC (Belczyński et al. 2000; Oliveira et al. 2013).

Jacoby SMC 7 has a period of $24.93 \pm 0.01$ days (Fig. 1). The object was disclaimed to be a genuine PN by Boroson \& Liebert (1989). It is located in an extended [O III] $5007 \AA$ and HI emission region of the supernova remnant (SNR) [FBR2002] J004806-730842. The spectrum of Jacoby SMC 7 contains H I lines, but the [O III] $5007 \AA$ emission was extracted with the diffuse background emission of the SNR (Fig. 3). Mennickent et al. (2002) observed a group of Be stars characterized by periods longer than 17 days. Jacoby SMC 7 may be a new member of this group.

Jacoby SMC 23 lies on the edge of an open cluster OGLE-CL SMC 104. The object experienced a sudden flux increase during the first season of the OGLE-III operation (Fig. 2). No variability was found before that event. No significant colour change accompanied the outburst. The object shows wide $\mathrm{H} \alpha$ line wings in the spectrum (Fig. 3), which were previously reported by Stanghellini et al. (2003). Jacoby SMC 23 is most likely a YSO experiencing an increase of the accretion 
M. Hajduk et al.: Search for binary central stars of the SMC PNe

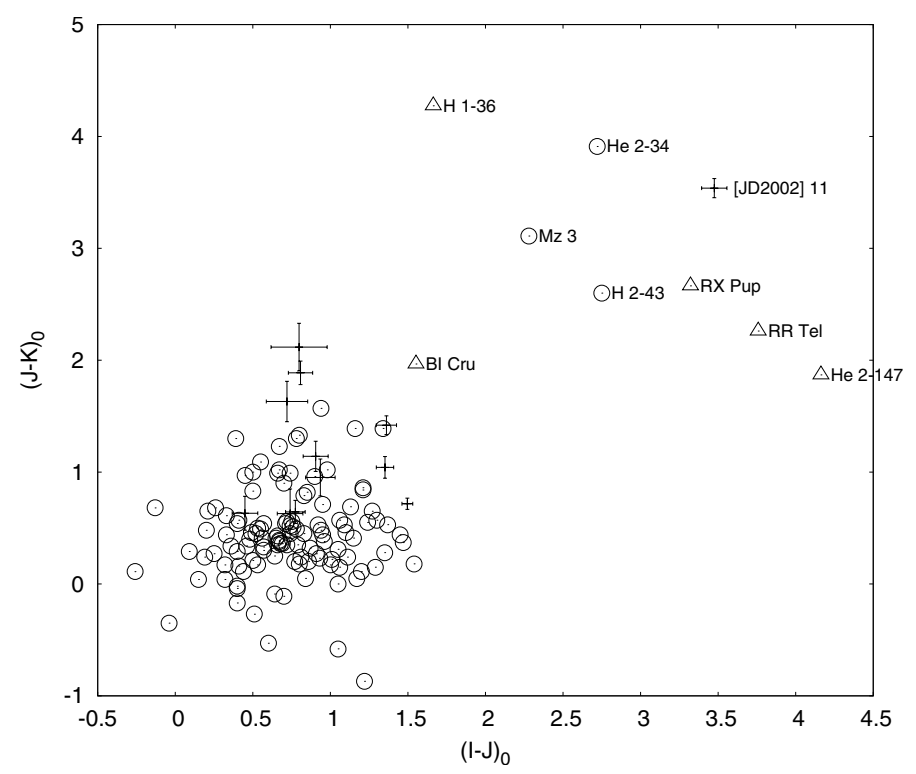

Fig. 4. IJK two-colour diagnostic diagram for the Galactic and SMC PNe. Open circles show Galactic objects classified as PNe, while triangles denote known symbiotic miras. Crosses denote the SMC PNe. Names of miras and suspected miras are labelled (Schmeja \& Kimeswenger 2001).

rate. It may be an optical counterpart of the YSO candidate S3MC J005530.39-725021.87 (Bolatto et al. 2007).

The spectrum of Jacoby SMC 24 shows only $\mathrm{H} \alpha$ and $\mathrm{H} \beta$ lines (Fig. 3). The light curve reveals irregular or semiregular variations (Fig. 2). We do not consider this object as a genuine PN. The nature of this PN candidate was previously debated by Morgan \& Good (1985) and the object was not recovered by Jacoby \& De Marco (2002). The light curve showing stochastic variability is similar to the light curves of most of the Be stars in the SMC (Mennickent et al. 2002).

LHA 115-N 61 shows eclipses lasting for three days with a period of about 138 days (Fig. 1). The light curve shows an additional 183-day variability (half a year) of instrumental origin (Fig. 2). There is no OGLE-II photometry of the source available since it was resolved by the pipeline. The object was suggested to be a diffuse nebula by Henize \& Westerlund (1963), because it was brighter than other $\mathrm{SMC} \mathrm{PNe}$ and resolved. The observed [O III] $5007 \AA / \mathrm{H} \beta$ flux ratio is of the order of unity, which may suggest a PN excited by a young central star. However, in that case the nebula would be compact and unresolved. The nebula is most likely excited by an eclipsing binary system of $\mathrm{O} V$ type stars. The depth of the eclipses may be supressed by the nebular contribution.

Jacoby SMC 1 is a binary CSPN with a period of $1.15539 \pm$ 0.00001 days assuming the irradiation efect to be the cause of the variations, or twice that period in the case of ellipsoidal variations (Fig. 1). The observed amplitude of the variations is about $0.3 \mathrm{mag}$, but may be supressed by the nebular contribution to the $I$ brightness. The spectrum, showing strong [O III] $5007 \AA$ emission and faint, blue continuum, strongly supports that the object is a genuine PN. However, we did not detect any irradiation lines coming from the cool component (Fig. 3), which suggests that the ellipsoidal effect is the cause of the variability.

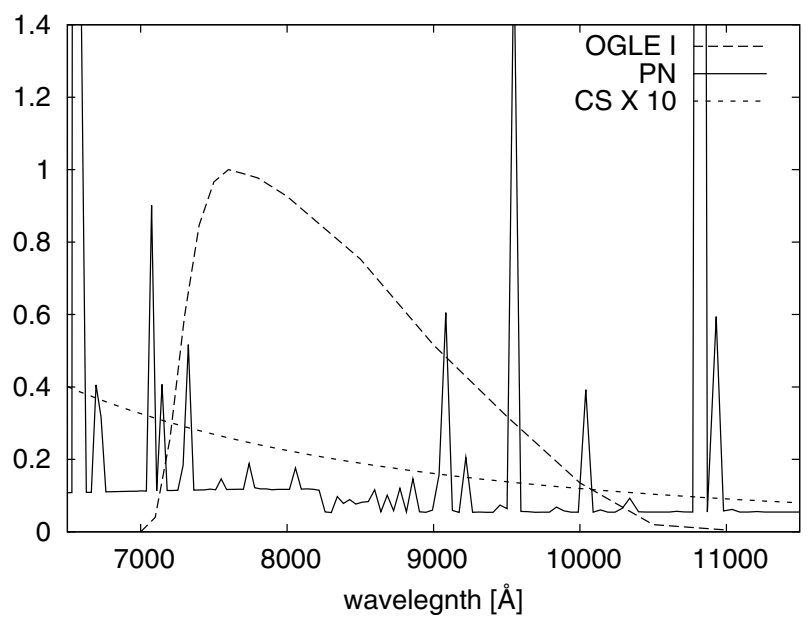

Fig. 5. OGLE I-band transmission curve and an example of PN and CS spectum.

\subsection{Binary fraction}

One binary CSPN was found in the SMC sample of $45 \mathrm{PNe}$. This suggests that only about $2 \%$ of SMC PNe may be the influenced by the CE phase, with the upper limit of about $7 \%$ (with $90 \%$ certainty, assuming $100 \%$ detection efficiency). This is lower than the binary fraction of 12 to $21 \%$ obtained for the Galactic bulge (Miszalski et al. 2009a). However, the observed binary fraction in the SMC is subject to small statictics and strong selection effects, some of which may be distance dependent. Below we list and assess the influence of the selection effects.

To determine the binary fraction, the sample must be first corrected for the PN mimics. As explained in Sect. 2, all the $\mathrm{PNe}$ in the sample were spectroscopically confirmed. Usually the SMC PNe were identified on the basis of the presence of the [O III] $5007 \AA$ line, lack of stellar continuum, and small angular dimensions (unresolved sources). Contamination of diffuse nebulae, Strömgren spheres in the interstellar medium, or compact $\mathrm{H}$ II regions is possible. About $10 \%$ of the sample show an [O III] $5007 \AA / \mathrm{H} \alpha$ flux ratio of the order of unity and may be unresolved diffuse nebulae.

Detection of photometric variations is possible only when the stellar continuum contributes significantly to the total brightness of the object in the $I$-band. The nebular lines and continuum can produce the main or even the entire contribution in the I-band in many spatially unresolved SMC PNe, supressing the amplitude of a binary central star. The HST observations showed that most of the SMC PNe do not exceed 1 arcsec in diameter (Stanghellini et al. 2003; Shaw et al. 2006) and would not be resolved in the OGLE images.

To assess the relative contribution of the stellar continuum to the total $I$-band flux of an unresolved PN, we used an exploratory PN model offered by the test suite distributed with the code Cloudy (Ferland et al. 1998). The model assumed that the $50000 \mathrm{~K}$ blackbody spectrum excites the ionization bounded nebula with reduced elemental abundances. The OGLE $I$-band transmission curve is shown along with the central star and nebular spectrum in Fig. 5. The [S III] 9069 and $9532 \AA$ lines contribute to the calculated brightness of the object in the nonstandard OGLE I-band filter (Udalski et al. 2002). The total nebular contribution is six time higher than the contribution of the central star in the $I$-band (18.6 mag at the distance to the SMC). The nebular contribution would be much lower for a cooler central star and a density bounded PN. 


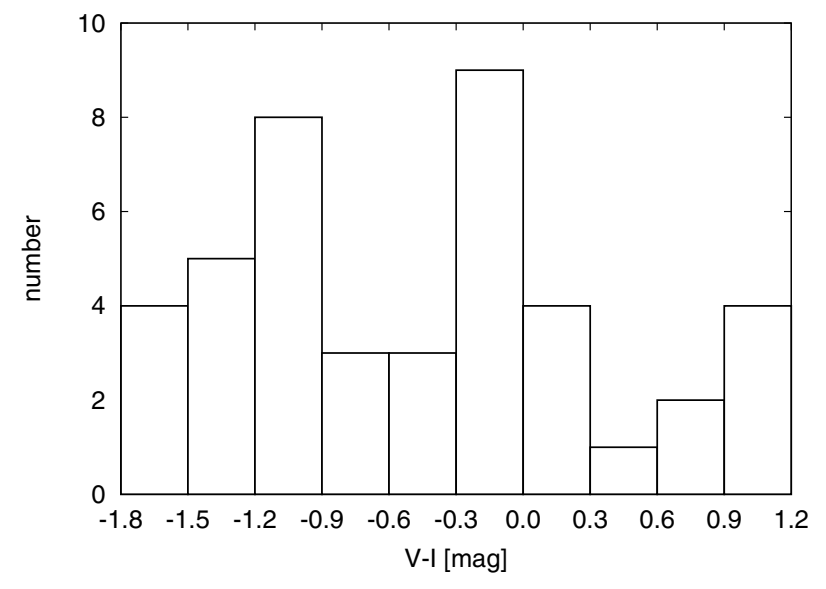

Fig. 6. Histogram of the $V-I$ colours of all identified SMC PNe.

We plot the histogram of the $V-I$ colours of the identified PNe in Fig. 6. Most of the objects have colours different from -0.4, expected for unreddened CSPN (Ciardullo et al. 1999), even those for which the central star was easily detected in the HST observations. The diagram is only slightly affected by reddening (an average $E(V-I)$ of 0.07 for the SMC, Haschke et al. 2011).

Central stars were detected in about $75 \%$ of the SMC PNe (Villaver et al. 2004). The nebular contribution could be significant in some of them. In $25 \%$ of cases no central star was detected, with the PN contributing all the light to the I-band. The inclusion of such objects in our sample can lower the derived binary fraction by about $25 \%$.

The SMC CSPNe are expected to be fainter than their Galactic bulge counterparts because of the difference in distance. This could lower the detection efficiency of the binary CSPNe. Assuming a similar intrinsic $I$-band brightness distribution of the SMC and the Galactic bulge CSPNe populations, the observed distributions would be separated by about 4.7 mag. However, Galactic bulge PNe are observed in the fields close to the Galactic centre, typically reddened by $E(V-I)$ of 1 or more (Nataf et al. 2013) contrary to the only slightly reddened SMC PNe (Haschke et al. 2011). In addition, SMC PNe are more likely to be unresolved and contribute to the $I$-band brightness. This would reduce the separation of both distributions.

The distributions of the $I$-band brightness of the SMC PNe and the Galactic bulge PNe known to harbour binary CSPNe (Miszalski et al. 2009a) are compared in Fig. 7. Bulge PNe are brighter on average only by about $1.5 \mathrm{mag}$ from the SMC PNe, and their brigntess distribution is much narrower. However, neither of the distributions can be treated as representative for the whole PN population. Very bright PNe, avoided by the OGLE-III pipeline, as well as very faint PNe may be underrepresented in the Galactic bulge histogram. Some of the SMC PNe fall below the completness limit of 21 mag for the OGLE-III survey in the I-band (Udalski et al. 2008).

The sensivity of our search depends on the apparent brightness of the object and the data sampling. Only one object in our sample has fewer than 100 datapoints. More than half of the PNe identified in the SMC fields are fainter than 18 mag (Fig. 7). However, the sensitivity limit for an 18 mag star observed 600 times is about 0.01 mag for a sinusoidal light curve. This is sufficient to detect most of the CSPNe with periods of up to 1-2 weeks, unless the binary system parameters were very unfavourable (De Marco et al. 2008). However, this requires at

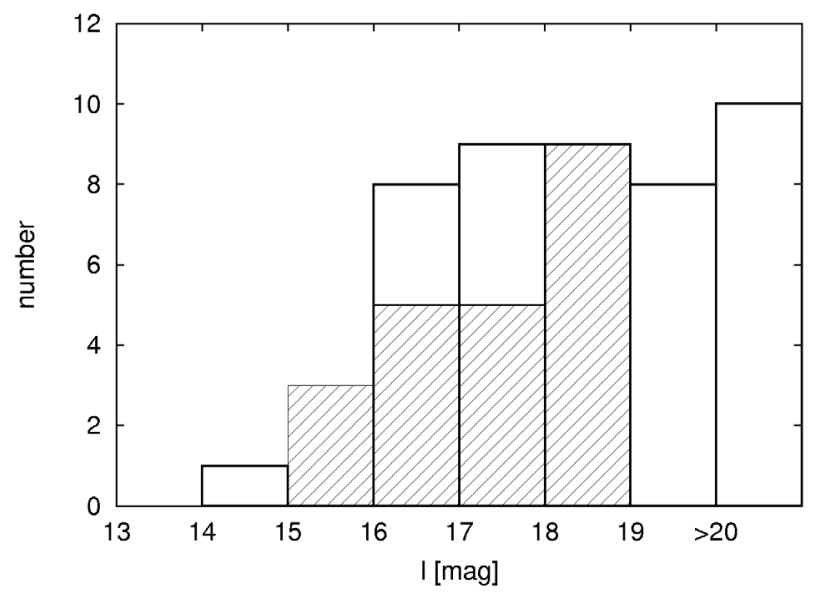

Fig. 7. Histogram of the $I$-band brightness of all identified SMC PNe. The hatched histogram of the binary CSPNe in the Galactic bulge is overplotted (Miszalski et al. 2009a).

least one of the binary components to be observable at the distance to the SMC.

An MV type companion of the CSPN would be below the OGLE detection limit at the distance to the SMC (fainter than 26 mag in the $I$-band). Binary CSPNe discovered purely because of irradiation effects constitute almost half of the systems detected by Miszalski et al. (2009a). A detection of the CSPN binaries showing ellipsoidal variations and eclipsing binaries would be feasible, although the detection efficiency would be reduced by the nebular contribution.

Taking all the selection effects into account, the number of detected binaries toward the SMC may be reduced by $80 \%$ compared with that of the Galactic bulge. The binary fraction corrected for the selection effects may be as high as $10 \%$ or higher.

\subsection{Period distribution}

The period distribution of the binary CSPNe in the Galaxy has a maximum of about 0.4 day with only few systems with periods longer than one day (Miszalski et al. 2011). The distribution of the close WD-MS CSPNe binaries appears to agree well with the WD-MS post CE systems (Hillwig 2011). The latter distribution shows a lack of systems with periods longer than 5-10 days (Nebot Gómez-Morán et al. 2011).

The period of Jacoby SMC 1 is three to six times longer than the maximum of the distribution for binary CSPNe in the Galaxy. The probability of finding of a CSPN binary with such a long period is relatively low, but not negligible. We cannot rule out the possibility that this reflects the real difference in the period distributions for the Galactic and SMC binary CSPNe.

We cannot confirm whether Jacoby SMC 1 contains a WD-MS or a double degenerate binary. The period distribution for the Galactic double degenerate binaries may have maximum shorter than 0.4 day.

The post-CE period distribution depends primarily on the binding parameter $\alpha_{\mathrm{CE}}$ and the initial mass-ratio distribution. The cutoff for the periods above a few days in the distribution of the Galactic binary CSPNe suggests the $\alpha_{\mathrm{CE}}=0.3$, otherwise a tail towards longer orbital periods of up to 1000 days would be expected (Miszalski et al. 2009a). The binding parameter of an envelope of a giant star does not show a strong dependance with metallicity (Loveridge et al. 2011), thus $\alpha_{\mathrm{CE}}$ may be equal to 0.3 for the SMC as well. 
The maximum of the period distribution depends on the assumed initial mass ratio. Zorotovic et al. (2011) have found that systems containing high-mass secondaries tend to have longer post-CE orbital periods for a Galactic sample for post-CE binaries. The amplitude of the photometric variability increases with the secondary mass, but decreases with the orbital separation. Thus binaries with high-mass secondaries may have similar amplitudes (and the detection efficiency) to the systems with lowmass companions. It is unclear, however, why would the SMC binaries have more massive secondaries.

Reduced mass loss during the AGB phase may lead to somewhat more massive central stars of PNe (Villaver et al. 2007), but no correlation between the WD mass and the periods of the post-CE systems was found for CO-core WDs (Zorotovic et al. 2011).

\section{Summary}

We discovered only one binary CSPN in the SMC. The actual binary fraction of the CSPNe in the SMC may be similar to the value obtained for the Galactic bulge PNe taking the selection effects into account. The sample is too small to derive firm conclusions concerning the period distribution of the post-CE binary CSPNe in the SMC.

In addition, observations allowed us to constrain the nature of some known PNe mimics. Three of them are probably Be stars and one is an YSO. The likelihood that [MA93] 22 is an optical counterpart of an Be/X-ray binary has increased. We discovered a new symbiotic star candidate.

Acknowledgements. This research has made use of the SIMBAD database, operated at CDS, Strasbourg, France. This work was financially supported by NCN of Poland through grants No. 2011/01/D/ST9/05966 and 719/N-SALT/2010/0. The OGLE project has received funding from the European Research Council under the European Community's Seventh Framework Programme (FP7/20072013)/ERC grant agreement no. 246678. Some of the observations reported in this paper were obtained with the Southern African Large Telescope (SALT), proposal 2012-1-POL-010, P.I. M. Hajduk. We thank the anonymous referee for the comments on the paper.

\section{References}

Belczyński, K., Mikołajewska, J., Munari, U., Ivison, R. J., \& Friedjung, M. 2000, A\&AS, 146, 407

Boffin, H. M. J., Miszalski, B., Rauch, T., et al. 2012, Science, 338, 773

Bolatto, A. D., Simon, J. D., Stanimirović, S., et al. 2007, ApJ, 655, 212

Bond, H. E. 2000, in Asymmetrical Planetary Nebulae II: From Origins to

Microstructures, eds. J. H. Kastner, N. Soker, \& S. Rappaport, ASP Conf. Ser., 199, 115

Boroson, T. A., \& Liebert, J. 1989, ApJ, 339, 844

Boyer, M. L., Srinivasan, S., van Loon, J. T., et al. 2011, AJ, 142, 103

Ciardullo, R., Bond, H. E., Sipior, M. S., et al. 1999, AJ, 118, 488

De Marco, O. 2009, PASP, 121, 316
De Marco, O. 2011, in Asymmetric Planetary Nebulae 5 Conference, Poster Session, held in Bowness-on-Windermere, UK, 20-25 June 2010, eds. A. A. Zijlstra, F. Lykou, I. McDonald, \& E. Lagadec, Jodrell Bank Centre for Astrophysics

De Marco, O., Hillwig, T. C., \& Smith, A. J. 2008, AJ, 136, 323

Evans, C. J., Howarth, I. D., Irwin, M. J., Burnley, A. W., \& Harries, T. J. 2004, MNRAS, 353, 601

Ferland, G. J., Korista, K. T., Verner, D. A., et al. 1998, PASP, 110, 761

Greenstein, J. L., \& Sargent, A. I. 1974, ApJS, 28, 157

Haberl, F., \& Sasaki, M. 2000, A\&A, 359, 573

Hajduk, M., Zijlstra, A. A., \& Gesicki, K. 2011, in Asymmetric Planetary Nebulae 5 Conference, Poster Session, held in Bowness-on-Windermere, UK 20-25 June 2010, eds. A. A. Zijlstra, F. Lykou, I. McDonald, \& E. Lagadec, Jodrell Bank Centre for Astrophysics

Haschke, R., Grebel, E. K., \& Duffau, S. 2011, AJ, 141, 158

Henize, K. G. 1956, ApJS, 2, 315

Henize, K. G., \& Westerlund, B. E. 1963, ApJ, 137, 747

Hillwig, T. C. 2011, in Asymmetric Planetary Nebulae 5 Conference, Poster Session, held in Bowness-on-Windermere, UK, 20-25 June 2010, eds. A. A. Zijlstra, F. Lykou, I. McDonald, \& E. Lagadec, Jodrell Bank Centre for Astrophysics

Jacoby, G. H. 1980, ApJS, 42, 1

Jacoby, G. H., \& De Marco, O. 2002, AJ, 123, 269

Jones, D., Pollacco, D., Faedi, F., \& Lloyd, M. 2011, in Asymmetric Planetary Nebulae 5 Conference, eds. A. Zijlstra, F. Lykou, I. McDonald, \& E. Lagadec, Jodrell Bank Centre for Astrophysics, 111P

Leisy, P., \& Dennefeld, M. 2006, A\&A, 456, 451

Lenz, P., \& Breger, M. 2005, Comm. Asteroseismol., 146, 53

Lindsay, E. M. 1961, AJ, 66, 169

Loveridge, A. J., van der Sluys, M. V., \& Kalogera, V. 2011, ApJ, 743, 49

Lutz, J., Fraser, O., McKeever, J., \& Tugaga, D. 2010, PASP, 122, 524

Mennickent, R. E., Pietrzyński, G., Gieren, W., \& Szewczyk, O. 2002, A\&A, 393, 887

Meyssonnier, N. 1995, A\&AS, 110, 545

Meyssonnier, N., \& Azzopardi, M. 1993, A\&AS, 102, 451

Miszalski, B., Acker, A., Moffat, A. F. J., Parker, Q. A., \& Udalski, A. 2009a, A\&A, 496, 813

Miszalski, B., Acker, A., Parker, Q. A., \& Moffat, A. F. J. 2009b, A\&A, 505, 249

Miszalski, B., Corradi, R. L. M., Jones, D., et al. 2011, in Asymmetric Planetary Nebulae 5 Conference, Poster Session, held in Bowness-on-Windermere, UK, 20-25 June 2010, eds. A. A. Zijlstra, F. Lykou, I. McDonald, \& E. Lagadec, Jodrell Bank Centre for Astrophysics

Morgan, D. H. 1995, A\&AS, 112, 445

Morgan, D. H., \& Good, A. R. 1985, MNRAS, 213, 491

Naik, S., Mathew, B., Banerjee, D. P. K., Ashok, N. M., \& Jaiswal, R. R. 2012, Res. Astron. Astrophys., 12, 177

Nataf, D. M., Gould, A., Fouqué, P., et al. 2013, ApJ, 769, 88

Nebot Gómez-Morán, A., Gänsicke, B. T., Schreiber, M. R., et al. 2011, A\&A, 536, A43

Oliveira, J. M., van Loon, J. T., Sloan, G. C., et al. 2013, MNRAS, 428, 3001

Sanduleak, N., \& Pesch, P. 1981, PASP, 93, 431

Sanduleak, N., MacConnell, D. J., \& Philip, A. G. D. 1978, PASP, 90, 621

Schmeja, S., \& Kimeswenger, S. 2001, A\&A, 377, L18

Schwarzenberg-Czerny, A. 1996, ApJ, 460, L107

Shaw, R. A., Stanghellini, L., Villaver, E., \& Mutchler, M. 2006, ApJS, 167, 201

Skrutskie, M. F., Cutri, R. M., Stiening, R., et al. 2006, AJ, 131, 1163

Stanghellini, L., Shaw, R. A., Balick, B., et al. 2003, ApJ, 596, 997

Udalski, A., Kubiak, M., \& Szymanski, M. 1997, Acta Astron., 47, 319

Udalski, A., Szymanski, M., Kubiak, M., et al. 2002, Acta Astron., 52, 217

Udalski, A., Soszyński, I., Szymański, M. K., et al. 2008, Acta Astron., 58, 329

Villaver, E., Stanghellini, L., \& Shaw, R. A. 2004, ApJ, 614, 716

Villaver, E., Stanghellini, L., \& Shaw, R. A. 2007, ApJ, 656, 831

Zorotovic, M., Schreiber, M. R., Gänsicke, B. T., et al. 2011, A\&A, 536, L3 